\title{
Combined therapy of temozolomide and ZD6474 (vandetanib) effectively reduces glioblastoma tumor volume through anti-angiogenic and anti-proliferative mechanisms
}

\author{
MI-YOUNG JO ${ }^{1,2^{*}}$, YOUNG GYU KIM ${ }^{6 *}$, YONGHYUN KIM ${ }^{1,2}$, SE JEONG LEE ${ }^{1,2,5}$, \\ MI HYUN KIM ${ }^{1,2}$, KYEUNG MIN JOO ${ }^{5}$, HYEON HO KIM ${ }^{3,4}$ and DO-HYUN NAM ${ }^{1,2}$ \\ ${ }^{1}$ Department of Neurosurgery, Samsung Medical Center and Sungkyunkwan University School of Medicine, Seoul; \\ ${ }^{2}$ Cancer Stem Cell Research Center, Samsung Biomedical Research Institute, Seoul; \\ ${ }^{3}$ Samsung Advanced Institute for Health Sciences and Technology, Sungkyunkwan University School of Medicine, Seoul; \\ ${ }^{4}$ Samsung Biomedical Research Institute, Samsung Medical Center, Seoul; ${ }^{5}$ Department of Anatomy, \\ Seoul National University College of Medicine, Seoul; ${ }^{6}$ Department of Neurosurgery, \\ Chungbuk National University College of Medicine, Chungbuk, Republic of Korea
}

Received February 20, 2012; Accepted March 28, 2012

DOI: $10.3892 / \mathrm{mmr} .2012 .868$

\begin{abstract}
Currently, clinically available options for treating glioblastoma (GBM) are quite limited, and there is a clear need to develop novel treatment strategies that can more effectively manage tumors. Here, we present a combination treatment of temozolomide (TMZ), a blood-brain barrier penetrating DNA alkylating agent, and ZD6474 (vandetanib), a VEGFR2 and EGFR dual-targeting anti-angiogenic agent, as a novel treatment strategy for GBM. In a U-87MG orthotopic xenograft model, the combination treatment provided a marked $94 \%$ tumor volume reduction. This reduction was greater than that achieved by monotherapy of either agent, and was correlated with a statistically significant reduction in microvessel density $\left(\mathrm{CD} 31^{+}\right.$cells) and proliferation $\left(\mathrm{PCNA}^{+}\right.$cells). These results confirm the necessity to target angiogenesis in addition to utilizing cytotoxic approaches, and provide the rationale for application of TMZ + ZD6474 combination therapy for treating GBM patients in the clinical setting.
\end{abstract}

Correspondence to: Professor Do-Hyun Nam, Department of Neurosurgery, Samsung Medical Center, Sungkyunkwan University School of Medicine, 50 Irwon-dong, Gangnam-gu, Seoul 135-710, Republic of Korea

E-mail: nsnam@skku.edu

Professor Hyeon Ho Kim, Samsung Advanced Institute for Health Science and Technology, Sungkyunkwan University School of Medicine, Seoul 135-710, Republic of Korea

E-mail: hyeonhkim@skku.edu

*Contributed equally

Key words: brain tumor, temozolomide, vandetanib, combination therapy

\section{Introduction}

Glioblastoma (GBM) is the deadliest and most malignant form of glioma. GBM presents as locally infiltrating and invasive types, and is associated with a poor prognosis due to the high proliferation of microvasculature $(1,2)$. Treatments for GBM typically involve surgical removal, radiation therapy and chemotherapy, but they often fail due to the highly resistant nature of the tumor $(3,4)$. Temozolomide (TMZ) is the most widely used chemotherapy for GBM and is orally administered (5). TMZ is a small-molecule DNA alkylating agent that easily penetrates the blood-brain barrier and elicits DNA methylation to effect tumor cell death $(6,7)$.

GBM is morphopathologically classified as having highly active neovascularization and proliferation, and there has been extensive interest in suppressing angiogenesis to control tumor growth. ZD6474 (vandetanib) is an anti-angiogenesis chemotherapeutic agent that is currently undergoing clinical trial for glioma (8) and non-small cell lung cancer (9). ZD6474 targets both vascular endothelial growth factor receptor (VEGFR) and epidermal growth factor receptor (EGFR), and is expected to perform better than existing anti-angiogenic therapeutics (9).

In this study, we studied the combination therapy of TMZ that suppresses tumor growth by DNA alkylation, together with ZD6474 whose anti-angiogenic mode of action is orthogonal to TMZ, in an orthotopic xenograft glioblastoma Balb/c-nu mouse model.

\section{Materials and methods}

Cell culture. The U-87MG human glioblastoma cell line was maintained in Eagle's minimal essential medium containing $1 \mathrm{mM}$ sodium pyruvate, $0.1 \mathrm{mM}$ non-essential amino acids, $2 \mathrm{mM}$ L-glutamine and $1.5 \mathrm{~g} / 1$ sodium bicarbonate, supplemented with $10 \% \mathrm{FBS}$ and $1 \%$ penicillin/streptomycin in standard tissue culture conditions. The cells were implanted 
into the mice within three passages for the development of the orthotopic xenograft models.

Orthotopic glioblastoma xenograft model generation. Six-week-old male Balb/c-nu mice were used. Mice were anesthetized with intramuscular injection of $100 \mathrm{mg} / \mathrm{kg}$ ketamine and $10 \mathrm{mg} / \mathrm{kg}$ xylazine and fastened to a stereotactic frame for the intracerebrum injection of U-87MG cells. U-87MG cells $\left(2 \times 10^{5}\right) / 5 \mu 1$ HBSS were injected using Hamilton syringes into the mice cerebrum at AP $0.5 \mathrm{~mm}, \mathrm{ML}+1.7 \mathrm{~mm}$, DV $-3.2 \mathrm{~mm}$ to generate the orthotopic glioblastoma xenograft model. The mice were subgrouped to control, TMZ alone, ZD6474 alone, and TMZ + ZD6474 combination groups. For the TMZ alone group, $2.5 \mathrm{mg} / \mathrm{kg}$ TMZ (Temodal ${ }^{\circledR}$ capsule; Schering-Plough, USA) was administered intraperitoneally on days 15-19 postU-87MG injection (Fig. 1). For the ZD6474 alone group, $50 \mathrm{mg} / \mathrm{kg}$ ZD6474 (vandetanib, 1751-100; BioVision, USA) was administered orally on days $15-28$ post-U-87MG injection (Fig. 1). For the combination group, TMZ and ZD6474 were administered using the same schedule as the respective single agent groups (Fig. 1). For all groups, mice were sacrificed at 4 weeks post-injection using $\mathrm{CO}_{2}$ gas. All animal experiments were performed according to the National Institute of Health Guide for the Care and Use of Laboratory Animals (NIH publication no. 80-23, revised in 1996), and according to the Association for Assessment and Accreditation of Laboratory Animal Care-Accredited Guidelines of our institute's Animal Use and Care Committee.

Immunohistochemistry. Brains collected from the mice were sectioned in 2-mm slices. The sections containing the injection site were fixed and embedded in $10 \%$ neutral formaldehyde, while the remaining sections were embedded in frozen blocks using OCT compound. Paraffin and frozen blocks were microtome sliced to 4 - and $8-\mu \mathrm{m}$ microslices, respectively, and stored at room temperature or $-20^{\circ} \mathrm{C}$, respectively. For tumor mass volume quantification, H\&E staining was performed in the paraffin sections and the sections were then observed under an optical microscope. Tumor volume was calculated as length $(\mathrm{mm}) \times$ [width $(\mathrm{mm})]^{2} \times 0.5$.

For CD31 staining, the frozen sections were dried at room temperature for $30 \mathrm{~min}$ and fixed in cold acetone, acetone:chloroform (1:1) and acetone (5 min each). After washing in PBS, the sections were incubated in $3 \% \mathrm{H}_{2} \mathrm{O}_{2} /$ methanol for 12 min to suppress the endogenous cellular peroxidase activity. After blocking in 5\% normal horse serum/1\% normal goat serum at room temperature for $20 \mathrm{~min}$, the sections were incubated in rat anti-mouse CD31 (BD Bioscience, 553370; 1:400 dilution) overnight at $4^{\circ} \mathrm{C}$ and washed three times in PBS. After reaction with horseradish peroxidase-conjugated anti-rat IgG for $1 \mathrm{~h}$, the sections were washed three times in PBS and developed in 3,3'-diaminobenzidine tetrahydrochloride (DAB-4HCl; Sigma, St. Louis, MO, USA). The sections were counterstained with Harris hematoxylin for $1 \mathrm{~min}$ and mounted for observation under an optical microscope. CD31-positive microvessels within the tumor were counted in randomly chosen, multiple fields of view.

For PCNA staining, paraffin was removed from sections using xylene, followed by multiple submersions in alcohol and distilled water for rehydration. The antigens were restored by

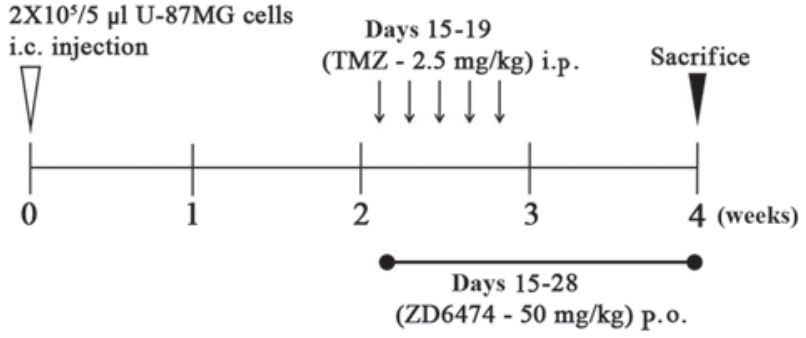

Figure 1. Experimental design. Six-week-old Balb/c-nu mice were used for generation of the orthotopic xenograft model. The control group did not receive any treatment. The TMZ alone group received $2.5 \mathrm{mg} / \mathrm{kg} \mathrm{TMZ}$ daily during days 15-19 post-injection. The ZD6474 alone group received $50 \mathrm{mg} /$ $\mathrm{kg}$ ZD6474 daily during days 15-28. The combination group received both agents at the same dose and schedule as the corresponding monotherapy group. All mice experiments were terminated on day 28. i.c., intracranial; i.p., intraperitoneal; p.o., per oral.

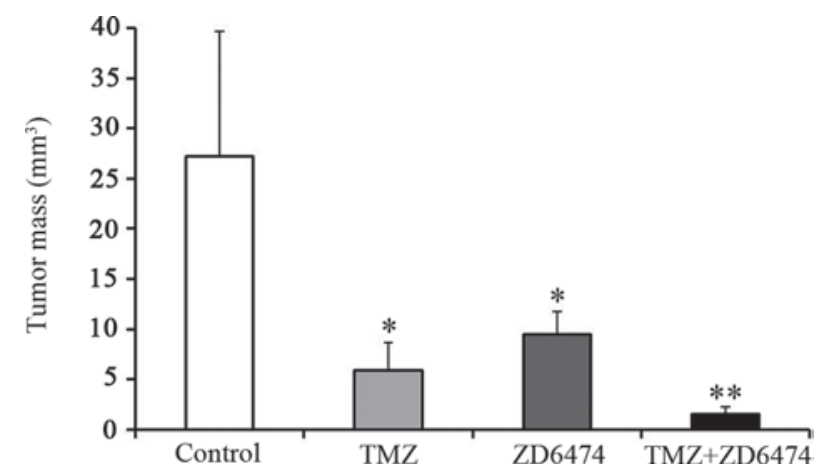

Figure 2. Synergistic effect of the combination treatment of TMZ and ZD6474 on tumor mass volume in the glioblastoma orthotopic xenograft model. Data are expressed as the means $\pm \mathrm{SE}$. ${ }^{*} \mathrm{p}<0.05,{ }^{* *} \mathrm{p}<0.01 \mathrm{vs}$. the control.

microwaving for $20 \mathrm{~min}$ in $0.1 \mathrm{M}$ citrate buffer. After washing in $\mathrm{PBS}$, endogenous peroxidase activity was suppressed as described above. To suppress non-specific staining, Mouse on Mouse kit (BMK-2202; Vector Laboratories, Burlingame, CA, USA) was used for $1 \mathrm{~h}$. Sections were incubated in PCNA primary antibody (M0879, Dako; 1:300 dilution) overnight at $4^{\circ} \mathrm{C}$. After washing with PBS, tissues were reacted with biotinconjugated anti-mouse secondary antibody (Vector Elite kit; Vector Laboratories) for $1 \mathrm{~h}$, followed by development in DAB $4 \mathrm{HCl}$ (Sigma). The sections were counterstained with Harris hematoxylin for $1 \mathrm{~min}$ and mounted for observation under an optical microscope. PCNA-positive cells within the tumor were counted in randomly chosen, multiple fields of view.

Statistical analyses. All results are reported as the means \pm standard error. One-way ANOVA was used for group-togroup comparison, followed by LSD test for multiple group comparison, with $\mathrm{p}<0.05$ denoting statistical significance.

\section{Results}

Synergistic effect of TMZ and ZD6474 on tumor volume reduction. To determine the synergistic effect of TMZ and ZD6474 combination therapy on glioblastoma, the tumor volume of the orthotopic xenograft models was measured. Twenty-eight 


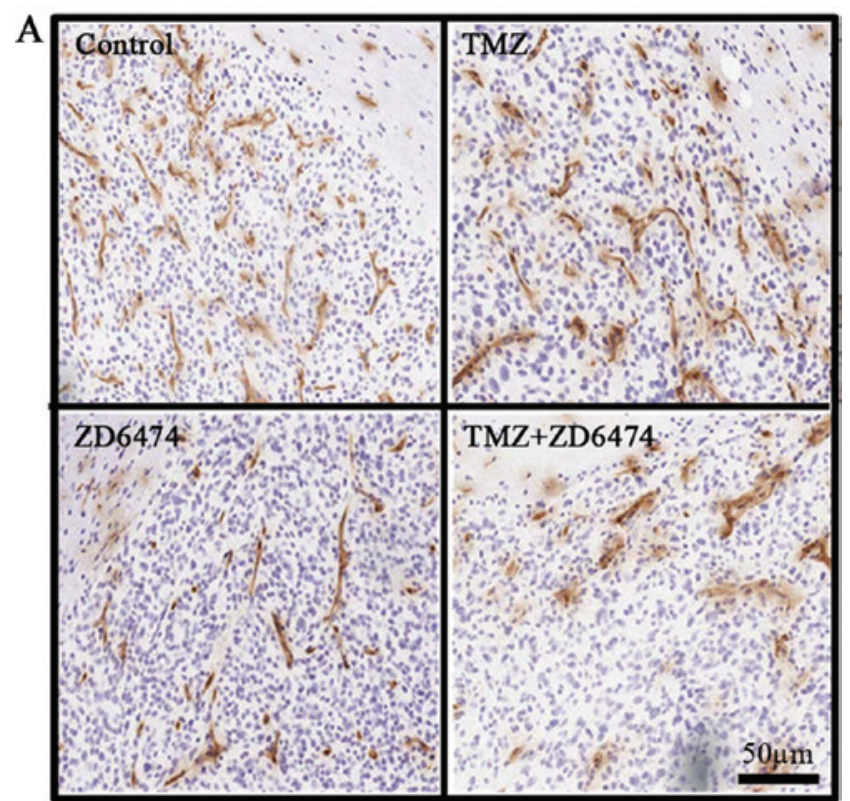

B

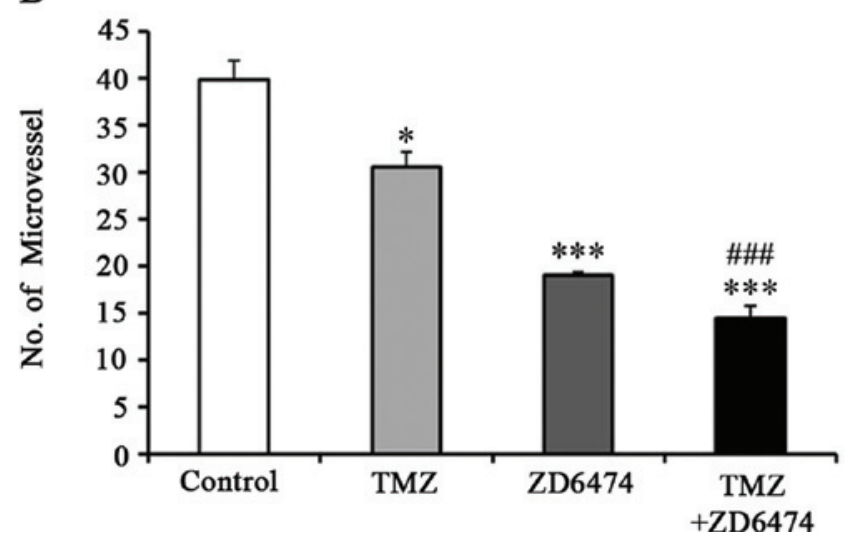

Figure 3. Synergistic effect of the combination treatment of TMZ and ZD6474 on microvessel density in the glioblastoma orthotopic xenograft model. (A) Immunohistochemistry with anti-CD31 in tumor tissue. (B) Mean numbers of microvessels (each group, $n=7$ ). Data are expressed as the means \pm SE. ${ }^{*}$ p $<0.05,{ }^{* * * *}$ p $<0.001$ vs. control; ${ }^{\# \# \# ~} \mathrm{p}<0.001$ vs. TMZ alone group

days after $\mathrm{U}-87 \mathrm{MG}$ intracerebrum injection, the control group exhibited a tumor volume of $27.2 \pm 12.4 \mathrm{~mm}^{3}$, while the TMZ and ZD6474 single agent treatment groups had significantly smaller tumor volumes of $5.9 \pm 2.8$ and $9.5 \pm 2.1 \mathrm{~mm}^{3}$, respectively $(\mathrm{p}<0.05)$. Furthermore, the TMZ and ZD6474 combination group exhibited even greater suppression of tumor growth, with a $1.6 \pm 0.7 \mathrm{~mm}^{3}$ tumor volume significantly different than that of the control $(\mathrm{p}<0.01)$. Compared to the single agent treatments, the combination treatment resulted in a $94 \%$ tumor suppression, confirming the synergistic effect of TMZ and ZD6474 (Fig. 2).

Synergistic effect of TMZ and ZD6474 on microvessel density reduction. There is a close correlative relationship between tumor growth and angiogenesis. Microvessel density (MVD) was determined by CD31 staining to evaluate the synergistic effect of the TMZ and ZD6474 combination therapy. The control group had a high MVD of $40 \pm 1.98$, while the TMZ

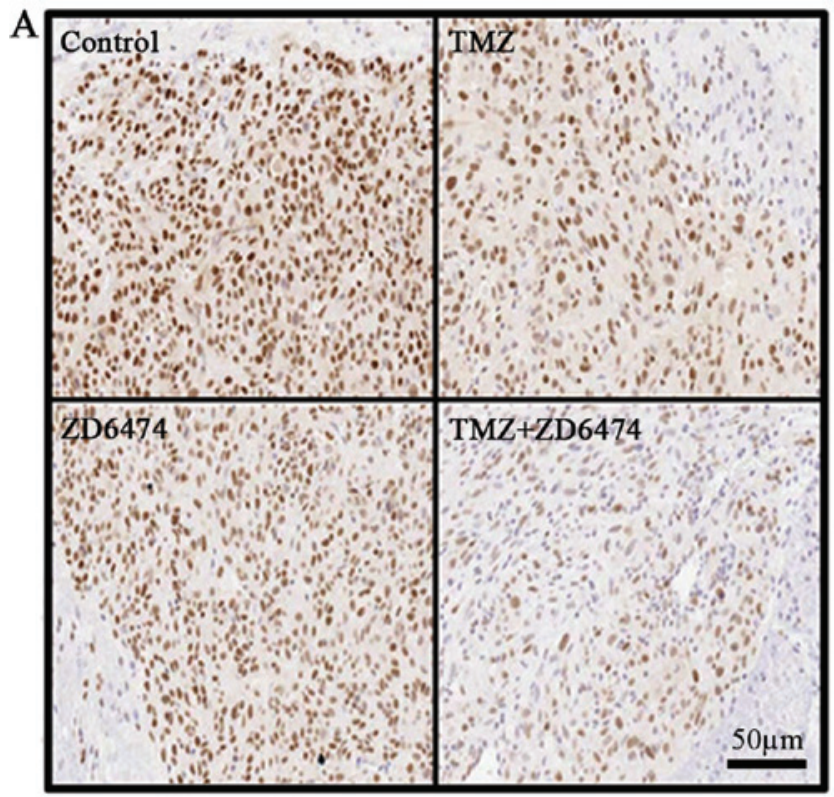

B

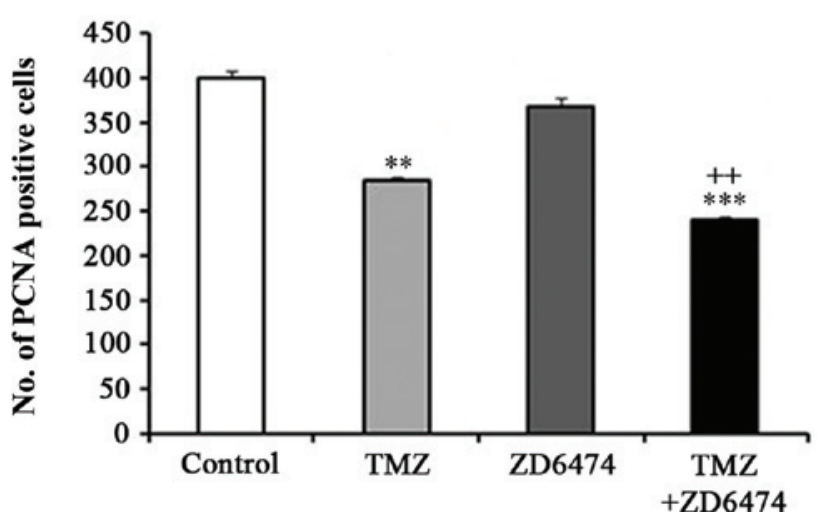

Figure 4. Synergistic effect of the combination treatment of TMZ and ZD6474 on cell proliferation in the glioblastoma orthotopic xenograft model. (A) Immunohistochemistry with anti-PCNA in tumor tissue. (B) Mean numbers of PCNA-positive cells (each group, $n=7$ ). Data are expressed as the means \pm SE. ${ }^{* *} \mathrm{p}<0.01,{ }^{* * *} \mathrm{p}<0.001$ vs. control; ${ }^{++} \mathrm{p}<0.01$ vs. ZD6474 alone group.

alone and ZD6474 alone groups had an MVD of $30 \pm 1.70$ and $19 \pm 0.28$, respectively, which were significantly different than the control $(\mathrm{p}<0.05$ and 0.001 , respectively). The TMZ + ZD6474 combination group exhibited an even greater MVD reduction of $14 \pm 1.32(\mathrm{p}<0.001)$, which correlated to a $57.7 \%$ MVD reduction $(\mathrm{p}<0.001)$ compared to the TMZ alone group, confirming the synergistic effect of the combination treatment (Fig. 3).

Synergistic effect of TMZ and ZD6474 on cell proliferation. Cell proliferation was characterized using PCNA staining. The control had an active proliferation count of $400 \pm 8$, while the TMZ alone and ZD6474 alone groups had 285 \pm 4 and $368 \pm 10$, respectively, showing a significant difference $(\mathrm{p}<0.01)$. The $\mathrm{TMZ}+$ ZD6474 combination group exhibited a greater significant reduction in the number of proliferating cells compared to the control $(\mathrm{p}<0.001)$, with a count of $240 \pm 4$. The reduction in the combination group was also statistically significant 
compared to the TMZ alone group $(\mathrm{p}<0.01)$, verifying that the combination treatment had a synergistic effect (Fig. 4).

\section{Discussion}

Glioblastoma (GBM) is the most malignant and lethal brain tumor. It is highly infiltrative to the surrounding tissues, such that total surgical resection is impossible, and additional radiotherapy and chemotherapy are imperative. Unfortunately, the median survival of GBM patients has not significantly improved (1-4). It has now become a standard to combine $\mathrm{TMZ}$ treatment with radiotherapy after surgical resection to help improve the survival of GBM patients. Yet, the median survival remains less than 2 years and novel therapeutic approaches, such as immunotherapy, gene therapy, antiangiogenic agents and growth factor inhibitors, are being pursued at the pre-clinical and clinical levels. Furthermore, to maximize the anticancer effects, combination therapy of two or more therapeutics with independent modes of action are being actively evaluated (10-12).

TMZ is administered orally because of its small molecular weight with lipophilic properties. It was developed as a DNA alkylating agent that directly damages tumor cells (5-7). TMZ is stable in acidic conditions, but breaks down to 5-(3-methyltriazen-1-yl)imidazole-4-carboxamide (MTIC) and forms methyldiazonium ions in alkaline conditions, such as the blood. It methylates the guanine base of the DNA to form $\mathrm{O}^{6}$ - and $\mathrm{N}^{7}$-methylguanine, leading the DNA doublestrand damage to induce apoptosis $(5-7,13)$. TMZ does not chemically cross-link with DNA and is known to have relatively minor toxicity to the hematopoietic system compared to other alkaline therapeutics. Its small size allows it to easily pass the blood-brain barrier and is therefore widely used in the clinic for the treatment of tumors of the central nervous system and metastatic melanoma $(14,15)$. Previous literature has reported that low dose, metronomic TMZ administration leads to lower resistance to the drug and even inhibits angiogenesis $(7,13)$. The present study also applied the low dose, metronomic TMZ therapy and observed a $80 \%$ reduction in tumor volume even with the single agent treatment.

To facilitate proliferative growth, tumor cells require an ample nutrient supply and thereby possess angiogenic potentials (16-18). Based on this observation, extensive development of anti-angiogenic agents that specifically reduce tumor cell growth has been carried out (16-18). Some of the factors known to be involved in angiogenesis include vascular endothelial growth factor (VEGF), epithelium growth factor (EGF), transforming growth factor (TGF), cathespin D, angiostatin, angiostatin-binding proteins, interleukin-4, VCAM-1 and cadherin, and the representative therapeutics in the clinic include thalidomide and ZD6474 (vandetanib) (16-18). For suppressing angiogenesis, therapeutics targeting EGFR have been developed since the receptor was shown to be involved in tumor growth, migration, infiltration and metastasis (19). ZD6474 is an orally administrable 4-anilinoquinazoline that specifically targets VEGFR2 and EGFR, and published reports indicate that its combination with radiotherapy leads to effective tumor control $(8,19)$. Based on these findings, we hypothesized that a combination of anti-angiogenic ZD6474 with TMZ would lead to a synergistic effect.
Our results show that both monotherapies (TMZ alone and ZD6474 alone) efficaciously reduced tumor volume, corroborating with previous reports $(7,8,13,20-22)$, while a more marked tumor volume reduction (94\%) was achieved with the combination treatment, due to the highly anti-angiogenic (64\% reduction of MVD) and anti-proliferative (40\% reduction) effects of the combined therapy. To further understand the mechanisms involved, future studies need to investigate the expression changes of angiogenic markers, such as VEGF and EGF. Evaluation of this combination therapy in other glioblastoma cell lines is also necessary $(23,24)$. Regardless, the study presented here provides the rationale for applying a combination therapy of TMZ and ZD6474 in the clinic in order to improve treatment efficacy compared to existing modalities for glioblastoma.

\section{Acknowledgements}

This study was supported by a grant of the Korea Healthcare Technology R\&D Project, Ministry for Health and Welfare Affairs, Republic of Korea (A092255).

\section{References}

1. Holland EC: Glioblastoma multiforme: the terminator. Proc Natl Acad Sci USA 97: 6242-6244, 2000.

2. Stupp R, Mason WP, van den Bent MJ, et al: Radiotherapy plus concomitant and adjuvant temozolomide for glioblastoma. N Engl J Med 352: 987-996, 2005.

3. Stupp R, Hegi ME, Gilbert MR and Chakravarti A: Chemoradiotherapy in malignant glioma: standard of care and future directions. J Clin Oncol 25: 4127-4136, 2007.

4. Van Meir EG, Hadjipanayis CG, Norden AD, Shu HK, Wen PY and Olson JJ: Exciting new advances in neuro-oncology: the avenue to a cure for malignant glioma. CA Cancer J Clin 60: 166-193, 2010.

5. Newlands ES, Stevens MF, Wedge SR, Wheelhouse RT and Brock C: Temozolomide: a review of its discovery, chemical properties, pre-clinical development and clinical trials. Cancer Treat Rev 23: 35-61, 1997.

6. Perry JR, Rizek P, Cashman R, Morrison M and Morrison T: Temozolomide rechallenge in recurrent malignant glioma by using a continuous temozolomide schedule: the 'rescue' approach. Cancer 113: 2152-2157, 2008.

7. Kim JT, Kim JS, Ko KW, et al: Metronomic treatment of temozolomide inhibits tumor cell growth through reduction of angiogenesis and augmentation of apoptosis in orthotopic models of gliomas. Oncol Rep 16: 33-39, 2006.

8. Sandstrom M, Johansson M, Bergstrom P, Bergenheim AT and Henriksson R: Effects of the VEGFR inhibitor ZD6474 in combination with radiotherapy and temozolomide in an orthotopic glioma model. J Neurooncol 88: 1-9, 2008.

9. Ryan AJ and Wedge SR: ZD6474 - a novel inhibitor of VEGFR and EGFR tyrosine kinase activity. Br J Cancer 92 (Suppl 1): 6-13, 2005 .

10. Gogas H, Polyzos A, Stavrinidis I, et al: Temozolomide in combination with celecoxib in patients with advanced melanoma. A phase II study of the Hellenic Cooperative Oncology Group. Ann Oncol 17: 1835-1841, 2006.

11. Kang SG, Kim JS, Park K, Groves MD and Nam DH: Combination celecoxib and temozolomide in C6 rat glioma orthotopic model. Oncol Rep 15: 7-13, 2006.

12. Son MJ, Kim JS, Kim MH, et al: Combination treatment with temozolomide and thalidomide inhibits tumor growth and angiogenesis in an orthotopic glioma model. Int J Oncol 28: 53-59, 2006.

13. Kong DS, Lee JI, Kim WS, et al: A pilot study of metronomic temozolomide treatment in patients with recurrent temozolomide-refractory glioblastoma. Oncol Rep 16: 1117-1121, 2006.

14. Agarwala SS and Kirkwood JM: Temozolomide, a novel alkylating agent with activity in the central nervous system, may improve the treatment of advanced metastatic melanoma. Oncologist 5: 144-151,2000. 
15. Seiter K, Katragadda S, Ponce D, Rasul M and Ahmed N: Temozolomide and cisplatin in relapsed/refractory acute leukemia. J Hematol Oncol 2: 21, 2009.

16. Jain RK: Normalization of tumor vasculature: an emerging concept in antiangiogenic therapy. Science 307: 58-62, 2005.

17. Bao S, Wu Q, Sathornsumetee S, et al: Stem cell-like glioma cells promote tumor angiogenesis through vascular endothelial growth factor. Cancer Res 66: 7843-7848, 2006.

18. Ono M, Izumi H, Yoshida S, et al: Angiogenesis as a new target for cancer treatment. Cancer Chemother Pharmacol 38: S78-S82, 1996.

19. Rich JN, Sathornsumetee S, Keir ST, et al: ZD6474, a novel tyrosine kinase inhibitor of vascular endothelial growth factor receptor and epidermal growth factor receptor, inhibits tumor growth of multiple nervous system tumors. Clin Cancer Res 11: 8145-8157, 2005.

20. Kong DS, Kim MH, Jeon JW, et al: Antitumor activity of ZD6474 in a metastatic orthotopic brain tumor model. Mol Med Report 1: 343-346, 2008
21. Ciardiello F, Bianco R, Caputo R, et al: Antitumor activity of ZD6474, a vascular endothelial growth factor receptor tyrosine kinase inhibitor, in human cancer cells with acquired resistance to antiepidermal growth factor receptor therapy. Clin Cancer Res 10: 784-793, 2004.

22. Sandstrom M, Johansson M, Andersson U, Bergh A, Bergenheim AT and Henriksson R: The tyrosine kinase inhibitor ZD6474 inhibits tumour growth in an intracerebral rat glioma model. Br J Cancer 91: 1174-1180, 2004.

23. Yiin JJ, Hu B, Schornack PA, et al: ZD6474, a multitargeted inhibitor for receptor tyrosine kinases, suppresses growth of gliomas expressing an epidermal growth factor receptor mutant, EGFRvIII, in the brain. Mol Cancer Ther 9: 929-941, 2010.

24. Drappatz J, Norden AD, Wong ET, et al: Phase I study of vandetanib with radiotherapy and temozolomide for newly diagnosed glioblastoma. Int J Radiat Oncol Biol Phys 78: 85-90, 2010. 